\title{
I criteri di ammissibilità probatoria
}

\author{
The evidence admission criteria
}

\section{Giulio Ubertis ${ }^{1}$}

Università Cattolica del Sacro cuore di Milano, Italia

giulio.ubertis@unicatt.it

http://orcid.org/0000-0003-0340-8143

\begin{abstract}
AвSTRACT: La verità giudiziale, presupposto per una decisione giusta, non può perseguirsi senza limiti. Le norme probatorie mirano a tutelare sia diritti soprattutto fondamentali sia criteri di razionalità processuale, rispettando la durata ragionevole del processo. La valutazione di ammissibilità probatoria ha esito positivo quando il tema di prova sia verosimile e pertinente, nonché qualora il mezzo e/o la fonte di prova siano rilevanti nel triplice senso della loro necessità (ossia, della loro non ridondanza), della loro idoneità contenutistica (perché atti a verificare l'enunciato da provare) e della loro idoneità epistemologica (in quanto l'attività compiuta sia conforme ai parametri gnoseologici storicamente dati). Si chiarisce infine che pure l'impiego dell'intelligenza artificiale deve garantire un controllo umano significativo.

Parole ChIAVE: Ammissione probatoria; Intelligenza artificiale; Pertinenza; Prova scientifica; Rilevanza; Verità giudiziale; Verosimiglianza.
\end{abstract}

ABSTRACT: Judicial truth, a prerequisite for a just decision, cannot be pursued without limits. Evidentiary norms aim to protect chiefly fundamental rights and the criteria of procedural rationality, respecting the reasonable time duration of the trial. The assessment of evidence submission is successful when the evidentiary theme is possible and pertinent, and where the means and / or sources of evidence are relevant in the threefold sense of their necessity (i.e. of their non-redundancy), of their content fitness (in that they can serve to verify the statement to be proved) and of their epistemological fitness (as the activity

1 Già Ordinario di Diritto processuale penale (Former Full Professor of Criminal procedure). 
performed complies with the historically given gnoseological parameters). Finally, it is clarified that the use of artificial intelligence must likewise guarantee meaningful human control.

KeYwords: Artificial intelligence; Evidence admission; Judicial truth; Pertinence; Relevance; Scientific evidence; Verisimilitude.

Sommario: 1. Verità e giustizia. - 2. Fondamenti delle norme probatorie e limiti temporali alla ricostruzione giudiziale. 3. La sequenza probatoria. - 4. La verosimiglianza e la pertinenza probatorie. -5 . La rilevanza probatoria: $a$ ) non ridondanza e idoneità contenutistica. - 6. b) idoneità epistemologica e "nuova" prova scientifica. - 7. Intelligenza artificiale e controllo umano significativo.

\section{VERITÀ E GIUSTIZIA}

Può considerarsi indiscusso che una decisione giurisdizionale sia reputata giusta solo quando sia fondata su una ricostruzione del fatto originante il processo ritenuta vera ${ }^{2}$. Ciò «indipendentemente dal criterio giuridico che si impiega per definire e valutare la giustizia della decisione» ${ }^{3}$, ossia dalla condivisione delle teorie procedurali o sostanziali di giustizia ${ }^{4}$ :

2 Sebbene, conformemente al linguaggio processualistico, il vocabolo "verità" - quando correlato a quelli di "ricerca", "accertamento" o simili - attenga essenzialmente alla ricostruzione del fatto, non vanno ignorate almeno due precisazioni: per un verso, non può escludersi un impiego del termine anche con riguardo alla soluzione del problema concernente l'individuazione della norma applicabile al caso concreto (cfr. FERRAJOLI Luigi, Diritto e ragione. Teoria del garantismo penale, Roma-Bari, 1989, p. 21 e passim); per l'altro, non può ignorarsi l'inevitabile connessione tra quaestio facti e quaestio iuris (v,. recentemente, UBERTIS Giulio, Quaestio facti e quaestio iuris, in Quaestio facti. Intern. J. Evid. Leg. Reas., 2020, n. 1, p. 69 ss.).

3 TARUFFO Michele, La prova dei fatti giuridici. Nozioni generali, in Trattato di diritto civile e commerciale, già diretto da CICU Antonio - MESSINEO Francesco e continuato da MENGONI Luigi, III, 2, 1, Milano, 1992, p. 43.

4 Una sintesi della loro differenza è esprimibile con il rilievo che, «mentre un atteggiamento si sforza di tenere distinti i problemi di natura politica, morale e giuridica, l'altro considera artificiale e inopportuna questa separazione» 
una base fattuale ritenuta erronea o inattendibile non potrebbe comunque supportare una decisione con la suddetta qualifica.

Conseguentemente, appare agevole riscontrare come la verità non sia in sé e per sé, lo scopo ultimo del processo, bensì - «compatibilmente con gli altri valori implicati dal medesimo» ${ }^{5}$ - il presupposto per poter convenientemente decidere quale sia la norma adatta alla vicenda su cui emettere la pronuncia.

Una conferma della connessione, ma altresì della distinzione, tra verità e giustizia risalta nella formula del giuramento contemplata per i giudici popolari nei giudizi di assise (art. 30 comma 11.10 aprile $1951 \mathrm{n}$. 287), dove sono scolpiti i requisiti necessari della sentenza, disponendo appunto che essa «riesca quale la società deve attenderla: affermazione di verità e di giustizia».

Quanto dunque attiene alla giurisdizione è una verità giudiziale, caratterizzata sia per la sua contestualità (cioè dipendenza dal sapere dato, pure metodologico, quando è perseguita, come accade in qualsivoglia settore di ricerca) sia per la sua funzionalità a quell'“obiettivo della giustizia” storicamente determinato dal vario comporsi dei valori presenti in mezzo al popolo nel cui nome (ai sensi dell'art. 101 comma 1 Cost.) la giustizia è amministrata ${ }^{6}$. È il parallelogrammo delle forze (individuali e collettive) interagenti nel processo a consentire una ricostruzione fattuale fondante una decisione "giusta" perché conforme a una verità che non assurge a meta ultima e assoluta dell'attività giurisdizionale: la sua conclusione ottiene il consenso dei cittadini anche con il rispetto della totalità delle opzioni ordinamentali durante l'intero sviluppo del procedimento.

A questo, assiologicamente e normativamente governato, interessa, per così dire, più il metodo del risultato: «la caccia vale più

(DAMAŠKA Mirjan R., I volti della giustizia e del potere. Analisi comparatistica del processo [1986], trad. it., Bologna, 1991, p. 129).

5 UBERTIS Giulio, Prova: II) teoria generale del processo penale, in Enc. giur. Treccani, Agg., XVII, Roma, 2009, p. 2.

6 UBERTIS Giulio, Fatto e valore nel sistema probatorio penale, Milano, 1979, p. 137. Analogamente, v. pure PERELMAN Ch., La preuve en droit, essai de synthèse, in La preuve en droit, a cura di Ch. Perelman - P. Foriers, Bruxelles, 1981, p. 364. 
della preda» $\left({ }^{7}\right)$ e «la giustizia della sentenza sta nel cammino seguito pel risultato» $\left({ }^{8}\right)$ o, meglio, il risultato che si consegue attraverso il processo è tale (hegelianamente espresso) «nel doppio senso di evento finale e di unità costituita dall'insieme degli altri eventi; i quali quindi ne sono e gli antecedenti e i momenti (aspetti particolari costitutivi)»?.

\section{FondAMENTI DELLE NORME PROBATORIE E LIMITI TEMPORALI ALLA RICOSTRUZIONE GIUDIZIALE}

In tema di ammissibilità (sebbene non solo rispetto a essa) emerge che il sistema probatorio si connota per essere il frutto di opzioni normative derivanti da due diversi ordini di motivi ${ }^{10}$, tra loro connessi e distintamente evidenziati per scopi di analisi.

Le regole probatorie legali, infatti, hanno un fondamento sia epistemologico (o processuale) che politico (o sostanziale): essi riguardano le esigenze, per il primo, di definire esplicitamente «un metodo per guidare il giudice nella ricerca» ${ }^{11}$ (come accade per gli 197, 214 comma 1, 403 e 499 commi 1-3 c.p.p.) e, per il secondo, di tutelare determinati diritti (si pensi agli art. 103 commi 2, 5 e 6, 199, 200, 201, 202, 219 comma 4 e 245 comma 2 c.p.p.), spesso costituzionalmente salvaguardati (ad

7 CORDERO Franco, Diatribe sul processo accusatorio (1965), in ID., Ideologie del processo penale, Milano, 1966, p. 220.

8 ASCARELLI Tullio, Processo e democrazia, in Riv. trim. dir. proc. civ., 1958, p. 858.

$9 \quad$ PRETI Giulio, Praxis ed empirismo, Torino, 1957, p. 170.

10 Per la loro classificazione, sostanzialmente analoga alla successiva esposizione, cfr. AMODIO Ennio, Libertà e legalità della prova nella disciplina della testimonianza, in Riv. it. dir. proc. pen., 1973, p. 326, 329; CAPPELLETTI Mauro, La testimonianza della parte nel sistema dell'oralità. Contributo alla teoria della utilizzazione probatoria del sapere delle parti nel processo civile, I, Milano, 1962, p. 283 ss., nota 1; CHIOVENDA Giuseppe, La natura processuale delle norme sulla prova e l'efficacia della legge processuale nel tempo (1912), in ID., Saggi di diritto processuale civile (1900-1930), I, Roma, 1930, p. 255 ss.; DENTI Vittorio, Scientificità della prova e libera valutazione del giudice, in Riv. dir. proc., 1972, p. 418; NUVOLONE Pietro, Le prove vietate nel processo penale nei paesi di diritto latino, ivi, 1966, p. 470.

11 CALAMANDREI Piero, Il giudice e lo storico, in Riv. dir. proc. civ., 1939, I, p. 115. 
esempio, dagli art. 2, 13, 14, 15, 19, 21, 24 e 32 Cost.). Tali prescrizioni possono corrispondere tanto a massime d'esperienza sedimentate nella coscienza giuridica ${ }^{12} \mathrm{o}$ a discrezionali scelte legislative quanto al generale patrimonio epistemologico storicamente dato.

In una prima prospettiva, le regole ammissive possono implicare divieti probatori, con cui si individuano modalità gnoseologiche incompatibili con l'ordinamento e pertanto si contemplano quelle che l'art. 190 comma 1 c.p.p. definisce «prove vietate dalla legge» (tipicamente riscontrabili negli art. 188 o 197 c.p.p.), talvolta collegandosi a impedimenti a compiere indagini su specifici argomenti (esemplificativamente, le «voci correnti nel pubblico» ex art. 194 comma 3 e 234 comma 3 c.p.p.) o a limitazioni negli strumenti di verifica attinenti a particolari temi (come avviene per la personalità dell'imputato, in riferimento alla quale possono acquisirsi i documenti inseriti nel catalogo dell'art. 236 c.p.p., ma, salve eccezioni, non può disporsi una perizia).

Relativamente alla sua derivazione da principi epistemologici, la disciplina probatoria è poi tendenzialmente immodificabile se questi non mutino: è ciò che accade per la garanzia del contraddittorio, non solamente inerente alla nozione di "giusto processo", ma pure basata sulla convinzione che «il metodo dialettico rappresenta finora quello migliore escogitato dagli uomini per stabilire la verità di enunciati fattuali, in qualsiasi campo e specialmente in quello giudiziario» ${ }^{13}$. Non a caso, «il legislatore penale contempla che, antecedentemente all'emanazione di qualsiasi ordinanza dibattimentale comportante il diniego di acquisizioni probatorie, sia attuato il contraddittorio tra le parti (art. 190 commi 1 e 3, 495 commi 1 e 4, 603 comma 5 c.p.p.), che potranno così contrastare la

12 Per il riconoscimento che la disciplina probatoria eleva spesso massime d'esperienza al rango di norma giuridica, v., ad esempio, CAPPELLETTI Mauro, Le grandi tendenze evolutive del processo civile nel diritto comparato (1968), in ID., Processo e ideologie, Bologna, 1969, p. 223-224; CHIOVENDA Giuseppe, L'oralità e la prova, in Riv. dir. proc. civ., 1924, I, p. 23; CORDERO Franco, Il procedimento probatorio, in ID., Tre studi sulle prove penali, Milano, 1963, p. 11-12; e, più anticamente, MITTERMAIER C.G.A., Teoria della prova nel processo penale (1834), trad. it., Milano, 1858, p. 101 ss.

13 UBERTIS Giulio, Ricostruzione del sistema, giusto processo, elementi di prova, in ID., Sisifo e Penelope. Il nuovo codice di procedura penale dal progetto preliminare alla ricostruzione del sistema, Torino, 1993, p. 268. 
riduzione dell'opera conoscitiva a vero e proprio 'cerimoniale tautologico' da cui venga rimossa la possibilità di conseguire risultanze gnoseologiche difformi dal 'desiderato'» ${ }^{14}$.

E il contradditorio si rivela tanto più necessario quanto più si osservi che le valutazioni concernenti l'ammissibilità probatoria sono dirette al vaglio delle descrizioni delle parti per rispettare i più elementari criteri di razionalità processuale: nonostante dal punto di vista logico «l'inferenza giudiziale non [sia] rigorosamente deduttiva ed esiste quindi un sia pur minimo margine d'incertezza o possibilità d'errore ... che in linea di principio [è innegabile] possa diminuire in seguito all'acquisizione di nuove prove» ${ }^{15}$, interest reipublicae ut sit finis litium e occorre conformarsi al canone della ragionevole durata del processo (art. 111 comma 2 Cost., 47 comma 2 Carta dir. fond. UE, 6 comma 1 Conv. eur. dir. uomo e 14 comma 3 lett. $c$ Patto intern. dir. civ. pol.), per cui viene tra l'altro in considerazione, come peculiare espressione di «una gigantesca regola controepistemica» ${ }^{16}$, la previsione di termini e modi nella presentazione delle richieste probatorie a opera delle parti (v., emblematicamente, art. 468 comma 1 c.p.p.).

A proposito dell'ammissibilità probatoria affiora quindi una seconda prospettiva di ricerca. Essa afferisce alla scelta di cosa introdurre nel bagaglio conoscitivo del giudice e pertanto alla costellazione concettuale delle nozioni, spesso confuse ${ }^{17}$, di verosimiglianza,

14 UBERTIS Giulio, Profili di epistemologia giudiziaria, Milano, 2015, p. 59. Si segnala che in tale volume sono già reperibili molte riflessioni esposte nel presente lavoro, ma di esso sarebbero poco eleganti citazioni ulteriori.

15 MURA Alberto, Teoria bayesiana della decisione e ragionevole durata del processo, in Cass. pen., 2007, p. 3105.

16 CAVALLONE Bruno, In difesa della veriphobia (considerazioni amichevolmente polemiche su un libro recente di Michele Taruffo) (2010), in ID., Scritti ritrovati sul processo civile e sul giudizio di fatto, Pisa, 2016, p. 274.

17 Cfr., per esempi di incompleta considerazione delle differenti nozioni o di confusione tra esse, sebbene nel contesto di alcuni tra i rari approcci definitori in materia, DENTI Vittorio, La verificazione delle prove documentali, Torino, 1957, p. 8; FERRUA Paolo, La prova nel processo penale, I, Struttura e procedimento, Torino, 2017, p, 122-123; FLORIAN Eugenio, Delle prove penali, I, In generale, Milano, 1924, p. 74; MELCHIONDA Achille, Prova in generale (diritto processuale penale), in Enc. dir., Agg., I, Milano, 1997, p. 843-844; MESSINA Salvatore, Il regime delle prove nel nuovo codice di procedura penale, Milano, 
pertinenza, rilevanza e concludenza probatorie, di cui occorre chiarire la portata semantica senza ignorare l'ausilio di "ridefinizioni" 18 , che non sono evitabili nemmeno per determinare il più esattamente possibile le molteplici accezioni della parola "prova", frequentemente adoperata in argomento (basti pensare alla locuzione "ammissione della prova") senza un'adeguata attenzione discretiva.

\section{LA SEQUENZA PROBATORIA.}

Il vocabolo prova può identificarsi in senso lato con quanto «destiné à établir une conviction sur un point incertain» ${ }^{19}$ riguardante la conoscenza del fatto all'origine della controversia giudiziaria, ossia con quell'insieme di elementi e attività, quel procedimento, quell'esito conoscitivo, aventi la funzione di consentire la verifica di uno degli enunciati fattuali integranti l'intero thema probandum.

Occorre tuttavia distinguere ciascuna delle componenti (spesso denominate "prova" senza specificazioni) strutturalmente costitutive della sequenza probatoria, le quali nel loro susseguirsi formano il fenomeno probatorio, analizzato, per così dire, in una prospettiva "statica".

Considerata la sua centralità gnoseologica, la prima e più importante di esse è l'elemento di prova, rappresentato da ciò che, introdotto nel procedimento, può essere utilizzato come premessa della successiva inferenza (per esempio: dichiarazione testimoniale, caratteristica dell'oggetto sequestrato, espressione contenuta in un documento, e così via).

1914, p. 16; TARUFFO Michele, Prova giuridica, in Enc. dir., Ann., I, Milano, 2007, p. 1030-1031.

«Si ha una ridefinizione quando, rimanendo ambito degli usi preesistenti, si determina in modo univoco e preciso il significato di una espressione, che in modo univoco e preciso non era usata. Si può estendere il nome di 'ridefinizione' anche al caso in cui si dà a una espressione un significato che essa non ha mai avuto, ma vicino al significato o alla gamma di significati che negli usi precedenti aveva. La ridefinizione è ... oggetto di una scelta, ma trae un carattere speciale dalla detta parentela con gli usi preesistenti» (SCARPELLI Uberto, Contributo alla semantica del linguaggio normativo [1959], Milano, 1985, p. 65-66).

19 LEVY-BRUHL Henri, La preuve judiciaire. Étude de sociologie juridique, Paris, 1964, p. 15. 
Il soggetto o l'oggetto da cui può derivare al procedimento almeno un elemento di prova è la fonte di prova, che a sua volta può essere tanto personale (come nel caso del teste) quanto reale (come nell'ipotesi del documento).

L'attività attraverso cui viene introdotto nel procedimento almeno un elemento di prova è il mezzo di prova (testimonianza, perquisizione, intercettazione telefonica, e così via $)^{20}$, per la cui esecuzione possono essere coinvolte più fonti di prova (si pensi ai soggetti che partecipano a un confronto o alle persone e alle cose impiegate per effettuare un esperimento giudiziale).

Sulla base dell'elemento di prova conseguito (o di più elementi di prova; per esemplificare, nell'eventualità che vengano reperite impronte digitali sull'arma del delitto, sarà necessario ottenere anche quelle dell'imputato per poter procedere all'analisi dattiloscopica di comparazione), si svolgerà il procedimento intellettivo (o, detto altrimenti, l'inferenza), il cui esito sarà rappresentato da una proposizione ${ }^{21}$ costituente il finale e propriamente denominabile risultato di prova (che, se reputato persuasivo dal giudice - perché, ad esempio, sono state da lui positivamente valutate l'affidabilità del testimone o l'autenticità del documento -, corrisponde alla conclusione probatoria fondativa della pronuncia ${ }^{22}$ ), eventualmente preceduto da uno o più passaggi, ciascuno

20 L'argomentata opinione che la differenziazione, sorta durante i lavori preparatori della riforma processuale del 1988, tra "mezzi di prova" e "mezzi di ricerca della prova" (poi trasfusa nei titoli II e III del libro III del codice di procedura penale) sia contraria all'esigenza di evitare l'uso di concetti superflui quando non addirittura oscuri, è stata recentemente ribadita con plurime argomentazioni da UBERTIS Giulio, Sistema di procedura penale, I, Principi generali, Milano, 2017, p. 83, nota 2.

21 "Proposizione", "enunciato" o "affermazione" sono termini (dei quali, d'altronde, manca un uso uniforme nella letteratura sul tema) che impiegheremo sinonimicamente pure per evitare fastidiose ripetizioni, considerato che ciò non incide sulle nostre considerazioni; invero, dovrebbe agevolmente risultare dal contesto se ci si riferisca all'espressione linguistica o al suo contenuto (tale distinzione si trova esemplificata in un lavoro di epistemologia giudiziaria da GARBOLINO Paolo, Probabilità e logica della prova, Milano, 2014, p. 20 ss.).

Emergono a questo proposito le due accezioni principali del termine "prova", occultate nel lessico italiano dalla mancanza di due vocaboli distinti: quella di esperimento gnoseologico (che nella lingua inglese verrebbe detto evidence) e quella di epilogo conoscitivo (che in inglese sarebbe la proof, ossia ciò che il giudice ritiene appreso a seguito dell'attività compiuta: v. EGGLESTON 
concludentesi con ciò che potrebbe altresì estensivamente chiamarsi risultato di prova, però "intermedio".

\section{LA VEROSIMIGLIANZA E LA PERTINENZA PROBATORIE}

Proprio perché inerente alla valutazione giudiziale sulla persuasività dello strumento conoscitivo ormai acquisito, in vista del suo impiego per il definitivo giudizio assertorio sulla verità dell'affermazione rappresentativa della res in iudicium deducta posta a base della domanda giudiziale, esula dai criteri di ammissibilità probatoria la nozione di concludenza, cui si è precedentemente alluso.

Con riferimento poi agli oggetti dei rispettivi giudizi, differiscono tra loro quelli dei giudizi di verosimiglianza e pertinenza, da un lato, e di rilevanza, dall'altro. Nonostante la loro comune funzione sia di delimitare l'attività istruttoria, verosimiglianza e pertinenza concernono gli enunciati da verificare, cioè i temi (od oggetti) di prova, mentre la rilevanza attiene alle fonti (si pensi a testimoni e documenti) e/o ai mezzi (come testimonianza, perizia, ispezione, perquisizione, ecc.) di prova.

Verosimiglianza e pertinenza, infatti, vertono «sulla affermazione del fatto, cioè sulla allegazione (positio) del fatto proveniente dalla parte che chiede di essere ammessa a provarlo» ${ }^{23} \mathrm{o}$ dall'autonoma attività di ricerca svolta dall'organo procedente (a seconda dell'assetto che ciascun sistema processuale riservi ai rapporti tra i poteri delle parti e quelli dell'autorità giurisdizionale per la formulazione dei temi di prova).

Relativamente al criterio di verosimiglianza, inoltre, gli oggetti di prova comunque individuati devono essere caratterizzati dalla loro ipotetica verificabilità, indispensabile per ogni orientamento assunto dall'indagine. Poiché «l'orizzonte istruttorio ha come limite l'epistemologia dominante» ${ }^{24}$,

Richard, Prova, conclusione probatoria e probabilità (1983), trad. it., Milano, 2004, spec. p. 191-192.

23 CALAMANDREI Piero, Verità e verosimiglianza nel processo civile, in Riv. dir. proc., 1955, I, p. 171.

24 CORDERO Franco, Procedura penale, Milano, 2012, p. 572, il quale, anziché l'aggettivo "verosimile", preferisce "probabile": ma, appunto, «in un senso diverso dal consueto, anche se morfologicamente esatto: 'quod probari possit'». 
viene richiesto al giudice di valutare se il fatto asserito non sia in contrasto con l'insieme delle leggi logiche e scientifiche non probabilistiche ${ }^{25} \mathrm{o}$, detto altrimenti, se possa essere accaduto secondo quello che è il patrimonio gnoseologico storicamente dato (non si richiamino, ad esempio, eventi magici per sostenere l'accadimento di un fatto $)^{26}$. Si tratta di un giudizio che afferisce all'aspetto specificamente storico dell'enunciato fattuale e che, non implicando un apprezzamento sulla plausibilità in concreto del tema di prova, non incorre in un'anticipazione della futura decisione, ma che va integrato da un'ulteriore disamina.

Poiché frustra probatur quod probatum non relevat, si deve ancora accertare se l'affermazione probatoria che si intende verificare è congruente con il thema decidendum.

25 Conviene peraltro rammentare che il legislatore può restringere il campo della ricerca processuale, richiamando in casi specifici pure la non contrarietà a massime d'esperienza, come succede con gli art. 2723 e 2724 n. 1 c.c. e 621 c.p.c., dove, in via di eccezione alle regole generali e riferendo l'aggettivo «verosimile» all'id quod plerumque accidit (cfr. TARUFFO Michele, La prova dei fatti giuridici. Nozioni generali, cit., p. 479), viene prima vietata e poi consentita solo parzialmente la prova testimoniale.

Conseguentemente, non sarebbe errato riservare il termine di "verosimiglianza" a questo caso e quello di "possibilità" a quello indicato nel testo (analogamente, v. SIRACUSANO Delfino, Studio sulla prova delle esimenti, Milano, 1959, p. 186-187).

Tuttavia, a livello epistemologico generale va condivisa l'ormai risalente osservazione di CARNELUTTI Francesco (Prova testimoniale di fatti inverosimili in materia di commercio, in Riv. dir. comm., 1923, II, p. 259), secondo cui non vi sarebbe «differenza, fuor da quella di grado, tra la impossibilità e la inverosimiglianza». E poiché ai nostri fini non pare sussistere l'esigenza di sceverare le due nozioni, si ritiene opportuno utilizzare unicamente il più tradizionale (cfr. CALAMANDREI Piero, Verità e verosimiglianza nel processo civile, cit., spec. p. 176; PUGLIATTI Salvatore, Conoscenza, in Enc. dir., IX, Milano, 1961, spec. p. 78) e onnicomprensivo termine di "verosimiglianza".

26 Suggestivamente, CORDERO Franco, Procedura penale, cit., p. 572, ipotizza che, se «un pubblico ministero occultista afferma che $\mathrm{N}$ abbia devastato le messi a P, scatenandogli sui campi fulmini e grandini, servizievolmente mandati da Satana, col quale aveva dei patti, e indica i relativi testimoni [, si avrebbe il compimento di] una mossa oziosa; l'assunto non costituisce tema proponibile ... [Ma] niente osterà a testimonianze o indagini peritali su simili 'maleficia', quando gli addetti al discorso scientifico riscoprano la categoria cosmologica del diabolico, elaborata dalla Scolastica, riaccreditando testi famosi come i Disquisitionum magicarum Libri sex del gesuita Martino del Rio»». 
Si tratta di applicare il criterio di pertinenza, in forza del quale attraverso un giudizio necessariamente inserito in coordinate giuridiche - si postula che l'ammissione probatoria possa essere disposta solo nel caso in cui si riconosca la sussistenza di un "nesso decisorio" (astrattamente individuato nel nuovo sistema processuale penale dalle classi di cui all'art. 187 c.p.p. ${ }^{27}$ ) tra singolo oggetto di prova e regiudicanda ${ }^{28}$ : è una valutazione $\mathrm{a}$ «carattere ipotetico nel senso che dà per provati i fatti [rectius: gli enunciati fattuali], di cui si chiede la prova, e si concentra nel controllare se sarebbero idonei a produrre le conseguenze giuridiche vagheggiate dalle parti ${ }^{29}$. Ma giova precisare come non occorra che il rapporto tra thema probandum e decidendum «sia diretto ed immediato, potendo essere soltanto e perfino mediato (così una circostanza da provare può essere pertinente per stabilire la credibilità di un teste)» ${ }^{30}$, quest'ultima evenienza inerendo all'ammissione di una cosiddetta "prova sussidiaria"31.

27 Né può sostenersi che ciò varrebbe unicamente per la fase giudiziale. Fin dai primi commenti al c.p.p. 1988, è stato chiarito che il suo art. 187 adempie alla funzione di «criterio-guida per lo sviluppo dell'attività probatoria ... [e] per la definizione dei suoi confini» (GREVI Vittorio, Libro III - Prove, in Profili del nuovo codice di procedura penale, diretti da CONSO Giovanni - GREVI Vittorio, Padova, 1990, p. 149) anche riguardo all'impiego di strumenti conoscitivi durante lo svolgimento delle indagini o dell'udienza preliminari. Altrimenti, il loro andamento sarebbe «ingovernabile» e la loro disciplina risulterebbe addirittura in gran parte «inapplicabile» (NOBILI Massimo, sub art. 187, in Commento al nuovo codice di procedura penale, coordinato da Chiavario Mario, II, Torino, 1990, p. 391).

${ }^{28}$ Sembra pertanto possibile considerare tale giudizio di pertinenza sostanzialmente affine a ciò che P. CALAMANDREI Piero, Verità e verosimiglianza nel processo civile, cit., 1955, I, p. 173, chiama «giudizio sulla rilevanza», ossia «un giudizio di diritto, attinente al merito, che contiene già, in nuce, la decisione definitiva», e che D. SIRACUSANO Delfino, Studio sulla prova delle esimenti, cit, p. 56-57, denomina «rilevanza in diritto ... determinata operando un collegamento con i fatti costitutivi ... [configuranti] i canoni che imprimono la prima direzione all'indagine e ne condizionano l'ulteriore svolgimento».

29 ANDRIOLI Virgilio, Prova (diritto processuale civile), in Nss. D.I., XIV, Torino, 1967, p. 273, dove peraltro è usato il vocabolo "rilevanza".

30 LEONE Giovanni, Trattato di diritto processuale penale, II, Svolgimento del processo penale. Il processo di prima istanza, Napoli, 1961, p. 181.

31 Così TARUFFO Michele, La prova dei fatti giuridici. Nozioni generali, cit., p. 431, designa il fenomeno relativo a una prova finalizzata alla valutazione di fonti e/o mezzi di prova. 
Ne deriva, in ottica giuridica, che, se tutto quanto è pertinente è pure verosimile, è scorretto l'enunciato contrario, non sempre conseguendo a un preliminare giudizio positivo di verosimiglianza quello di pertinenza.

Ma non va ignorato come ambedue dipendano dal momento processuale in cui vengano formulati: invero, se un'eventuale richiesta probatoria, anche per scongiurare attività superflue, va respinta quando «esprime delle circostanze già sufficientemente accertate ... [ovvero] ritenute inidonee a dare un nuovo indirizzo» alla ricerca ${ }^{32}$, «l'esperibilità di nuove indagini diminuisce progressivamente col procedere l'accertamento verso la decisione» ${ }^{33}$ : e in proposito basti ricordare il restrittivo parametro della "assoluta necessità", per il legislatore processuale penale costituente requisito per consentire "nuove" acquisizioni probatorie ai sensi degli art. 507 e 523 comma 6 c.p.p.

\section{LA RILEVANZA PROBATORIA: A) NON RIDONDANZA E IDONEITÀ CONTENUTISTICA}

Il momento processuale nel quale viene formulato assume importanza pure per il giudizio di rilevanza probatoria, che però attiene alla delimitazione non degli oggetti, bensì delle fonti e/o dei mezzi di prova, di cui bisogna acclarare dapprima la necessità. Occorre che le fonti e/o i mezzi di prova siano non ridondanti, poiché non sarebbero acquisibili quando non assolvessero a una funzione diversa dal ribadire ciò che fosse già stato conseguito con prove precedenti (od ottenibile con successive, se il giudizio di rilevanza fosse compiuto in un unico contesto con riferimento a più richieste e si decidesse di accoglierne solo alcune).

Ma, per essere rilevanti, tali fonti e/o mezzi, devono altresì caratterizzarsi, rispetto alla verifica di un tema di prova ritenuto verosimile e pertinente, per la loro idoneità ${ }^{34}$, sussistente anzitutto in una prospettiva

32 SIRACUSANO Delfino, Studio sulla prova delle esimenti, cit., p. 190.

33 SIRACUSANO Delfino, Studio sulla prova delle esimenti, cit., p. 189.

34 Pure VERDE Giovanni, Prova in generale: b) teoria generale e diritto processuale civile, in Enc. dir., XXXVII, Milano, 1988, p. 620, parla di «giudizio di rilevanza ... [come] valutazione di idoneità del mezzo di prova». Mentre, proseguendo il confronto con altrui terminologie, può individuarsi una affinità tra 
contenutistica (per un altro profilo, v. il successivo paragrafo): essendo cioè atte a veicolare elementi di prova da cui inferire proposizioni in grado di confermare o smentire l'affermazione probatoria cui intendano rapportarsi. Si esige quindi un apprezzamento in concreto sulla congruenza tra quanto conseguibile in sede probatoria e l'enunciato che si intenda verificare, così da impedire uno sterile dispendio di tempo quando già si sappia che l'esperimento gnoseologico avrebbe un esito non fruibile pro o contra nessuna delle parti; né viene implicata un'anticipazione del compito decisorio, poiché si tratta di una valutazione vertente non sull'affidabilità, attendibilità o credibilità (cioè, comunque sulla forza persuasiva) dell'operazione probatoria ${ }^{35}$, ma sulla assoluta inutilità di quest'ultima per la sua incapacità a fornire elementi di prova di cui potersi servire in sede deliberativa nel controllo dell'oggetto di prova considerato. Per esemplificare, sarebbe irrilevante per mancanza di idoneità contenutistica una ricognizione vocale, qualora sin dal momento della richiesta probatoria fosse nota e non contestata la totale sordità congenita della persona chiamata a effettuarla.

la nozione di "rilevanza" che proponiamo e ciò che D. SIRACUSANO (Studio sulla prova delle esimenti, cit., p. 58) e TARUFFO Michele (Studi sulla rilevanza della prova, Padova, 1970, p. 33), definiscono, rispettivamente, «rilevanza in fatto» e «rilevanza in senso stretto», non avendo adoperato il termine "pertinenza" per effettuare lessicalmente una differenziazione con la "rilevanza". Una simile distinzione, invece, viene ad esempio proposta da MELCHIONDA Achille, Prova in generale (diritto processuale penale), cit., p. 843-844, nonché (sebbene con esiti diversi da quelli illustrati nel testo, almeno perché ambedue le nozioni vengono riferite a uno stesso «dato probante», senza poi chiarire se questo vada inteso come oggetto, elemento, fonte, mezzo o risultato di prova) da SABATINI Giuseppe, Prova (diritto processuale penale e diritto processuale penale militare), in Nss. D.I., XIV, Torino, 1967, p. 318.

35 Sembrano incorrere in tale fraintendimento (criticando l'impostazione già sostenuta in UBERTIS Giulio, La prova penale. Profili giuridici ed epistemologici, Torino, 1995, p. 62-63) sia DOMINIONI Oreste, La prova penale scientifica. Gli strumenti scientifico-tecnici nuovi o controversi e di elevata specializzazione, Milano, 2005, p. 222, sia PAULESU Pier Paolo, Giudice e parti nella "dialettica" della prova testimoniale, Torino, 2002, spec. p. 124 e nota 55, dove si richiama, quale esempio giurisprudenziale sulla valutazione di richieste probatorie (erroneamente) ipotizzata come coincidente con il criterio delineato nel testo, un provvedimento relativo all'(in)attendibilità di testimoni per il loro interesse a deporre in un senso o in un altro. 


\section{B) IDONEITÀ EPISTEMOlOgicA E "NUOVA" PROVA SCIENTIFICA}

Il sistema processuale impone tuttavia che la rilevanza della fonte e/o del mezzo di prova da assumere possegga un'idoneità anche epistemologica, per l'esigenza che l'elemento (ipoteticamente) conoscitivo sia conseguito o manipolato in modo tale da permettere il controllo delle parti e della collettività sull'esercizio della giurisdizione e sulla sua conformità al principio di legalità processuale (cfr., per i singoli aspetti qui sintetizzati, art. 1 comma 2, 101, 102 comma 3, 111 commi 1 e 6 Cost.). Siccome difformi dai parametri epistemologici attuali e pertanto inidonei all'impiego nella trama logico-argomentativa del provvedimento $^{36}$, non possono avere ingresso nel processo attività dirette a verificare un tema di prova verosimile e pertinente, ma ispirate, per esemplificare, alla magia, all'oracolarità, alla rabdomanzia, allo spiritismo, alla grafologia ${ }^{37}$.

È comprensibile come l'importanza di tale caratteristica sia emersa soprattutto con riguardo alla cosiddetta nuova prova scientifica, con cui nell'ambito dei «mezzi di prova nei quali si usa uno strumento scientificotecnico che richiede specifiche competenze e quindi l'intervento di un esperto» ${ }^{38}$ - si denominano le operazioni probatorie utilizzanti apparati tecnico-scientifici reputati di generalmente elevata specializzazione e nuovi

36 Per analoga soluzione, v. CORDERO Franco, Procedura penale, cit., p. 615; DOMINIONI Oreste, La prova penale scientifica. Gli strumenti scientifico-tecnici nuovi o controversi e di elevata specializzazione, cit., p. 99-101.

Quest'ultima, intesa come studio della scrittura in quanto reputata rivelatrice del carattere, va distinta dall'analisi grafica volto a verificare se un atto sia autentico, cui si riferisce l'art. 75 norme att. c.p.p.

DOMINIONI Oreste, Prova scientifica (diritto processuale penale), in Enc. dir., Ann., II, 1, Milano, 2008, p. 977. Una nozione "allargata" di prova scientifica è invece proposta da GIUNTA Fausto, Questioni scientifiche e prova scientifica tra categorie sostanziali e regole di giudizio, in Prova scientifica, ragionamento probatorio e decisione giudiziale, a cura di BERTOLINO Marta UBERTIS Giulio, Napoli, 2015, p. 57, includendovi «tutti gli accertamenti condotti sulla base di un sapere specialistico, ancorché non tecnologico, comunque estraneo alla formazione professionale del giurista» e pertanto assimilando in un'unica classe ciò che il legislatore distingue quando, ad esempio, prevede la perizia se occorrano «specifiche competenze tecniche, scientifiche o artistiche» (art. 220 comma 1 c.p.p.). 
o controversi ${ }^{39}$ : giova quindi chiarire che la questione può concernere pure la prova scientifica, per così dire, tradizionale (riferita a conoscenze comunemente condivise al momento del loro uso nel processo e per le quali l'eventuale «problematicità dell'accertamento probatorio si concentra ... non sulla premessa nomologica, ma sul suo corretto impiego pratico» ${ }^{40}$ ), quando ne fosse posta in dubbio la «reale 'scientificità'» ${ }^{41}$ in seguito allo sviluppo degli studi che la concernano.

Il richiamo alla scienza, infatti, non è sufficiente a tacitare il timore che siano inserite nel quadro gnoseologico componenti di dubbio valore scientifico (la cosiddetta "scienza spazzatura") per la preoccupazione di tralasciare indagini magari determinanti nella soluzione dei casi giudiziari. È stata così sostenuta in ambito penale la proposta, attraverso «un'interpretazione analogica» ${ }^{42}$ dall'art. 189 c.p.p. (disciplinante la prova atipica), di interpretare il sistema processuale penale in maniera da consentire in Italia comportamenti analoghi a quelli seguiti nel mondo anglosassone, dove è attribuita al giudice la scelta di procedere a un apprezzamento caso per caso sulla forza persuasiva della "nuova prova scientifica" prima di deciderne l'ammissione ${ }^{43}$.

Tale impostazione, però, si scontra con alcuni ostacoli insormontabili.

In una prospettiva epistemologica, si assegna al giudice la responsabilità di assumere una posizione talvolta estremamente difficile (e

39 DOMINIONI Oreste, La prova penale scientifica. Gli strumenti scientifico-tecnici nuovi o controversi e di elevata specializzazione, cit., p. 13. Anche la Corte europea dei diritti dell'uomo ha da tempo riconosciuto il particolare valore di siffatti strumenti, invitando i giudici nazionali a tenerne conto (C.edu, sez. III, sent. 9 novembre 2006, Tavli c. Turchia, § 36).

40 GIUNTA Fausto, Questioni scientifiche e prova scientifica tra categorie sostanziali e regole di giudizio, cit., p. 63.

41 CAPRIOLI Francesco, La scienza "cattiva maestra": le insidie della prova scientifica nel processo penale, in Cass. pen., 2008, p. 3529.

42 DOMINIONI Oreste, In tema di nuova prova scientifica, in Dir. pen. proc., 2001, p. 1062, mentre F. CAPRIOLI, La scienza "cattiva maestra": le insidie della prova scientifica nel processo penale, cit., p. 3528, specifica che «soltanto» una siffatta esegesi consentirebbe «l'applicazione dell'art. 189 c.p.p. alla prova scientifica 'nuova'».

43 DOMINIONI Oreste, La prova penale scientifica. Gli strumenti scientifico-tecnici nuovi o controversi e di elevata specializzazione, cit., spec. p. 102 ss. 
forse impossibile) da giustificare e non rientrante nelle sue competenze, per poter decidere se seguire le usuali regole dell'ammissione probatoria o quelle speciali della "nuova prova scientifica". Egli dovrebbe cioè selezionare ciò che non è incontestabilmente distinguibile, dato che «il dato acquisito al dibattito epistemologico, pure nelle sue proiezioni giuridico-processuali, per cui la scienza non è in grado di dare certezza incontrovertibili, non consente di tracciare una sicura e netta linea di demarcazione fra 'conoscenze unanimemente accertate' e conoscenze che tali non sono» ${ }^{44}$, appunto perché nuove (e conseguentemente non ancora sottoposte a un adeguato collaudo dagli studiosi della materia) o controverse.

In ottica giuridica, la soluzione ipotizzata, anzitutto, confligge con il principio di legalità processuale (statuito dall'art. 111 comma 1 Cost. e individuato tra i principi generali del diritto dalla Corte europea dei diritti dell'uomo ${ }^{45}$ ) per il richiamo all'analogia, per di più con il fine di applicare il regime della prova atipica (della cui legittimità costituzionale si dubita ${ }^{46}$ ).

Inoltre, affidare al giudice il compito di formulare «un giudizio di prevalutazione dell'attendibilità o dell'efficacia della prova $»^{47}$ violerebbe i requisiti di imparzialità, terzietà e, più specificamente, neutralità metodologica del giudice, poiché «non può ammettersi che l'organo giurisdizionale proceda alla verifica delle affermazioni delle parti tenendo comportamenti che siano influenzati da una delle prospettazioni inerenti alla regiudicanda o ne sottintendano l'accoglimento ${ }^{48}$. Non a caso, nell'esperienza statunitense presa a modello ${ }^{49}$, diversi sono i soggetti

44 DOMINIONI Oreste, La prova penale scientifica. Gli strumenti scientifico-tecnici nuovi o controversi e di elevata specializzazione, cit., p. 369. C.edu, sez. II, sent. 22 giugno 2000, Coëme e altri c. Belgio, § 102.

CARRATTA Antonio, Prova e convincimento del giudice nel processo civile, in Riv. dir. proc., 2003, p. 55.

47 DOMINIONI Oreste, La prova penale scientifica. Gli strumenti scientifico-tecnici nuovi o controversi e di elevata specializzazione, cit., p. 231. UBERTIS Giulio, Sistema di procedura penale, I, Principi generali, cit., p. 146.

49 Essa è ampiamente illustrata da DOMINIONI Oreste, La prova penale scientifica. Gli strumenti scientifico-tecnici nuovi o controversi e di elevata specializzazione, cit., p. 116 ss. 
(rispettivamente, il giudice togato e la giuria) che esprimono la valutazione sull'ammissibilità probatoria e quella sul merito della regiudicanda, sebbene resti poi da giustificare la sottrazione dell'apprezzamento di persuasività probatoria di uno strumento conoscitivo al giudice chiamato a decidere sulla regiudicanda (ciò che in Italia parrebbe confliggere almeno con gli art. 25 comma 1 e 101 comma 2 Cost.). «La diversità tra i procedimenti di ammissione italiano e nordamericano, però, non esclude che vengano valorizzati anche nel primo i parametri emersi nella giurisprudenza del secondo e finalizzati all'esclusione dal processo della pseudo-scienza ${ }^{50}$, ulteriormente raffinati dalla Cassazione senza ignorare l'esigenza di verificare la competenza dell'esperto del cui parere ci si intenda avvalere ${ }^{51}$.

Infine, riguardo al rito penale (e senza ignorare ulteriori più specifiche considerazioni ${ }^{52}$ ), questo già contiene norme in cui sarebbero agevolmente inquadrabili le innovazioni tecnico-scientifiche, in relazione alle quali varrebbe l'ordinaria disciplina dell'art. 190 c.p.p.: si pensi alle ricognizioni di «voci, suoni o ... quanto altro può essere oggetto di ricognizione sensoriale» (art. 216 comma 1 c.p.p.), alle perizie ex art. 220 ss. c.p.p., per le quali «la natura particolare ed inconsueta degli accertamenti non vale a determinarne la traslazione al novero delle prove 'atipiche' $»^{53}$, o ai documenti, tra cui è annoverabile, oltre agli scritti, tutto quanto rappresenti «fatti, persone o cose mediante la fotografia, la cinematografia, la fonografia o qualsiasi altro mezzo» (art. 234 comma 1 c.p.p.).

Dunque, pure per la "nuova prova scientifica" va attribuita all'organo giurisdizionale l'esecuzione non di un giudizio anticipato sulla sua affidabilità, attendibilità o credibilità, bensì (come per ogni altra ipotesi di ammissione probatoria) di una stima sull'idoneità per lo strumento utilizzato

50 UBERTIS Giulio, Prova scientifica e giustizia penale, in Riv. it. dir. proc. pen., 2016, p. 1202.

51 V., ad esempio, Cass., sez. IV, 29 gennaio 2013, Cantore, in Giust. pen., 2013, II, c. 695 (in motivazione); Cass., sez. IV, 17 settembre 2010, Cozzini, in Cass. pen., 2011, p. 1701 (in motivazione).

52 Cfr. RENZETTI Silvia, La prova scientifica nel processo penale: problemi e prospettive, in Riv. dir. proc., 2015, p. 411-412; UBERTIS Giulio, Il giudice, la scienza e la prova (2011), in ID., Argomenti di procedura penale, IV, 2016, p. 29 ss.

53 Cass., sez. I, 21 maggio 2008, Franzoni, in Cass. pen., 2009, p. 1860 (in motivazione). 
a conseguire un esito concretamente fruibile (anche perché razionalmente controllabile e giustificabile) per la verifica del singolo enunciato costituente il tema di prova. L'eventuale controversia sulla scientificità della prova richiesta sarebbe risolubile altresì attraverso lo sviluppo del contraddittorio nel suo momento ammissivo, magari usufruendo di prove sussidiarie per accertare controllabilità e giustificabilità dei procedimenti e dei risultati relativi all'apparato tecnico-scientifico: andrebbe comunque «evita[to] di addossare al proponente l'onere di provare la rilevanza di quanto richiesto, garantendogli l'ammissione dell'esperimento conoscitivo, salva la sua irrilevanza» ${ }^{54}$, nel dovuto rispetto dell'art. 190 c.p.p.

Solo nel successivo momento valutativo il giudice - dotato non di una cultura scientifica "di merito", ma di una "cultura di criteri, consistente in schemi concettuali intesi a scrutinare la validità delle leggi scientifiche e delle tecnologie usate dall'esperto e la loro corretta applicazione" 55 - potrà argomentativamente dire se l'esperimento gnoseologico ha effettivamente adempiuto o no la sua funzione di convincimento e ha quindi conclusivamente consentito il formarsi, sulla sua base, di un epilogo conoscitivo «comprensibile da chiunque, conforme a ragione ed umanamente plausibile ${ }^{56}$.

\section{INTELLIGENZA ARTIFICIALE E CONTROLLO UMANO SIGNIFICATIVO}

Il valore delle conclusioni fin qui raggiunte non è da porre in dubbio nemmeno rispetto a un argomento che sempre più viene affrontato in ambito giudiziario: l'impiego in esso della cosiddetta intelligenza artificiale, la cui complessità di profili non può essere affrontata in questa sede e la cui stessa novità rende difficile fornirne una nozione univoca.

Un minimo livello di consenso è forse ottenibile riconoscendo quali caratteristiche principali dell'intelligenza artificiale «a) l'uso di

54 Linee guida per l'acquisizione della prova scientifica nel processo penale, in Scienza e processo penale: linee guida per l'acquisizione della prova scientifica, a cura di DE CATALDO NEUBURGER Luisa, Padova, 2010, p. 5.

${ }_{55}$ DOMINIONI Oreste, La prova penale scientifica. Gli strumenti scientifico-tecnici nuovi o controversi e di elevata specializzazione, cit., p. 71.

56 Cass., sez. IV, 29 gennaio 2013, Cantore, cit., c. 695 (in motivazione). 
grandi quantità di dati e informazioni; b) una elevata capacità logicocomputazionale; c) l'uso di nuovi algoritmi, come quelli del deep learning e dell'auto-apprendimento, che definiscono metodi per estrarre conoscenza dai dati per dare alle macchine la capacità di prendere decisioni corrette in vari campi di applicazione» ${ }^{57}$, senza escludere una modifica degli algoritmi originari «man mano che ricevono più informazioni su quello che stanno elaborando» ${ }^{58}$.

Inoltre, giova riprendere e adattare al contesto giudiziario la proposta, sorta durante il dibattito internazionale sviluppatosi nell'ambito dell'ONU sulle armi autonome ( pilota), di spostare l'attenzione dalla forse insuperabile difficoltà di una loro definizione alla «necessità di assicurare che gli attacchi sferrati da tutti i sistemi d'arma siano soggetti a un 'controllo umano significativo'» ${ }^{59}$.

Dopo aver rammentato la regola generale sull'osservanza dei diritti fondamentali, con la conseguenza che non sarebbe mai ammissibile una cosiddetta prova digitale se violatrice di essi (il principio del nemo tenetur se detegere, ad esempio, potrebbe essere leso da una profilazione personologica ${ }^{60}$ ), occorre perciò evidenziare che ostacolo all'utilizzabilità della prova è la sua «opacità» ${ }^{61}$. Come già altrove sostenuto ${ }^{62}$, andrebbe

57 MAGRO Maria Beatrice, Robot, cyborg e intelligenze artificiale, in Cybercrime, diretto da CADOPPI Alberto - CANESTRARI Stefano - MANNA Adelmo -PAPA Michele, Milano, 2019, p. 1181.

58 G. SIMEONE, Machine Learning e tutela della Privacy alla luce del GDPR, in Diritto e intelligenza artificiale, a cura di ALPA Guido, Pisa, 2020, p. 280, nota 15.

59 TAMBURRINI Guglielmo, Etica delle macchine. Dilemmi morali per robotica e intelligenza artificiale, Roma, 2020, p. 105.

60 MANES Vittorio, L'oracolo algoritmico e la giustizia penale: al bivio tra tecnologia e tecnocrazia, in Intelligenza artificiale. Il diritto, i diritti, l'etica, a cura di RUFFOLO Ugo, Milano, 2020, p. 563.

${ }^{61}$ QUATTROCOLO Serena, Quesiti nuovi e soluzioni antiche? Consolidati paradigmi normativi vs rischi e paure della giustizia digitale "predittiva", in Cass. pen., 2019, p. 1764, dove ricorda che «non è consentito che nella funzione probatoria si usino apparati conoscitivi insuscettibili di controllo ad opera del giudice e delle parti» (O. DOMINIONI, La prova penale scientifica. Gli strumenti scientifico-tecnici nuovi o controversi e di elevata specializzazione, cit., p. 69).

62 Si ripropone qui recentissima opinione espressa in UBERTIS Giulio, Intelligenza artificiale, giustizia penale, controllo umano significativo, in Sistema penale, 11 novembre 2020, p. 14-15, consultabile all'indirizzo internet 
pertanto sancito che l'impiego della "macchina" (secondo la convenzionale denominazione abbreviata del "sistema [o sinonimicamente: dell'apparato] di intelligenza artificiale") in sede giurisdizionale fosse assoggettato a un controllo umano significativo costituito dalle seguenti imprescindibili condizioni: 1) pubblicità e vaglio conforme ai criteri di peer review riguardo al suo funzionamento; 2) informazione sul potenziale tasso di errore ${ }^{63}$; 3) adeguate spiegazioni traducenti la "formula tecnica" rappresentativa dell'algoritmo nella sottesa regola giuridica, in tal modo resa leggibile e comprensibile dal giudice, dalle parti e dai loro difensori ${ }^{64}$; 4) garanzia del contraddittorio sulla scelta degli elementi archiviati, sui loro raggruppamenti e sulle correlazioni dei dati elaborati dall'apparato di intelligenza artificiale, specialmente con riferimento all'oggetto della controversia; 5) giustificazione da parte del giudice del loro accoglimento alla luce di quanto emerso in giudizio e valutato secondo il principio del libero convincimento.

Il controllo umano significativo, dunque, andrebbe altresì garantito qualora, come prospettato ${ }^{65}$, l'intelligenza artificiale fosse impiegata al momento dell'ammissione probatoria, in cui non dovrebbe mancare, rispetto all'uso della macchina, il suo apprezzamento e la corrispondente motivazione da parte del giudice.

\section{BibLiografiA}

AMODIO Ennio, Libertà e legalità della prova nella disciplina della testimonianza, in Riv. it. dir. proc. pen., 1973, p. 310-339

https://sistemapenale.it (ultimo accesso il 13 gennaio 2021), cui si rinvia per ulteriori indicazioni bibliografiche.

${ }^{63}$ Appare indubbia l'ispirazione dei precedenti criteri a quelli della famosa sentenza Daubert, il cui tetralogo è sinteticamente esposto da CARLIZZI Gaetano, La valutazione della prova scientifica, Milano, 2019, p. 91, e da RIVELLO Pier Paolo, La prova scientifica, in Trattato di procedura penale, diretto da UBERTIS Giulio - VOENA Giovanni Paolo, XVIII, Milano, 2014, p. 79-80.

64 Similmente, v. Cons. Stato, sez. VI, 5 dicembre 2019 - 4 febbraio 2020 n. 881, MIUR c. Barra e Rosolia, § 10, consultabile all'indirizzo internet www. giustizia-amministrativa.it (ultimo accesso il 13.1.2021).

65 NIEVA-FENOLL Jordi, Intelligenza artificiale e processo (2018), trad. it., Torino, 2019, p. 93 ss. 
ANDRIOLI Virgilio, Prova (diritto processuale civile), in Nss. D.I., XIV, Torino, UTET, 1967, p. 260-300

ASCARELLI Tullio, Processo e democrazia, in Riv. trim. dir. proc. civ., 1958, p. 844-860

CALAMANDREI Piero, Il giudice e lo storico, in Riv. dir. proc. civ., 1939, I, p. 105-128

CALAMANDREI Piero, Verità e verosimiglianza nel processo civile, in Riv. dir. proc., 1955, I, p. 164-192

CAPPELLETTI Mauro, La testimonianza della parte nel sistema dell'oralità. Contributo alla teoria della utilizzazione probatoria del sapere delle parti nel processo civile, I, Milano, Giuffrè, 1962

CAPPELLETTI Mauro, Le grandi tendenze evolutive del processo civile nel diritto comparato (1968), in ID., Processo e ideologie, Bologna, il Mulino, 1969, p. 169-251

CAPRIOLI Francesco, La scienza "cattiva maestra": le insidie della prova scientifica nel processo penale, in Cass. pen., 2008, p. 3520-3538

CARLIZZI Gaetano, La valutazione della prova scientifica, Milano, Giuffrè, 2019

CARNELUTTI Francesco, Prova testimoniale di fatti inverosimili in materia di commercio, in Riv. dir. comm., 1923, II, p. 255-265

CARRATTA Antonio, Prova e convincimento del giudice nel processo civile, in Riv. dir. proc., 2003, p. 27-64

CAVALLONE Bruno, In difesa della veriphobia (considerazioni amichevolmente polemiche su un libro recente di Michele Taruffo) (2010), in ID., Scritti ritrovati sul processo civile e sul giudizio di fatto, Pacini, Ospedaletto, 2016, p. 245-279.

CHIOVENDA Giuseppe, La natura processuale delle norme sulla prova e l'efficacia della legge processuale nel tempo (1912), in ID., Saggi di diritto processuale civile (1900-1930), I, Roma, Foro Italiano, 1930, p. 241-259

CHIOVENDA Giuseppe, L’oralità e la prova, in Riv. dir. proc. civ., 1924, I, p. 5-32 CORDERO Franco, Diatribe sul processo accusatorio (1965), in ID., Ideologie del processo penale, Milano, Giuffrè,1966, p. 201-232

CORDERO Franco, Il procedimento probatorio, in ID., Tre studi sulle prove penali, Milano, Giuffrè, 1963, p. 3-144

CORDERO Franco, Procedura penale, Milano, Giuffrè, 2012 
DAMAŠKA Mirjan R., I volti della giustizia e del potere. Analisi comparatistica del processo [1986], trad. it., Bologna, il Mulino, 1991

DENTI Vittorio, La verificazione delle prove documentali, Torino, UTET, 1957

DENTI Vittorio, Scientificità della prova e libera valutazione del giudice, in Riv. dir. proc., 1972, p. 414-437

DOMINIONI Oreste, La prova penale scientifica. Gli strumenti scientifico-tecnici nuovi o controversi e di elevata specializzazione, Milano, Giuffrè, 2005

DOMINIONI Oreste, Prova scientifica (diritto processuale penale), in Enc. dir., Ann., II, 1, Milano, Giuffrè, 2008, p. 976-998

EGGLESTON Richard, Prova, conclusione probatoria e probabilità (1983), trad. it., Milano, Giuffrè, 2004

FERRAJOLI Luigi, Diritto e ragione. Teoria del garantismo penale, Laterza, Roma-Bari, 1989

FERRUA Paolo, La prova nel processo penale, I, Struttura e procedimento, Torino, Giappichelli, 2017

FLORIAN Eugenio, Delle prove penali, I, In generale, Milano, Vallardi, 1924

GARBOLINO Paolo, Probabilità e logica della prova, Milano, Giuffrè, 2014

GIUNTA Fausto, Questioni scientifiche e prova scientifica tra categorie sostanziali e regole di giudizio, in Prova scientifica, ragionamento probatorio e decisione giudiziale, a cura di BERTOLINO Marta - UBERTIS Giulio, Napoli, Jovene, 2015, p. 55-84

GREVI Vittorio, Libro III - Prove, in Profili del nuovo codice di procedura penale, diretti da CONSO Giovanni - GREVI Vittorio, Padova, CEDAM, 1990, p. 139-192

ISISC (Istituto Superiore Internazionale di Scienze Criminali), Linee guida per l'acquisizione della prova scientifica nel processo penale, in Scienza e processo penale: linee guida per l'acquisizione della prova scientifica, a cura di DE CATALDO NEUBURGER Luisa, Padova, CEDAM, 2010, p. 3-13

LEONE Giovanni, Trattato di diritto processuale penale, II, Svolgimento del processo penale. Il processo di prima istanza, Napoli, Jovene, 1961

LEVY-BRUHL Henri, La preuve judiciaire. Étude de sociologie juridique, Paris, Rivière, 1964

MAGRO Maria Beatrice, Robot, cyborg e intelligenze artificiale, in Cybercrime, diretto da CADOPPI Alberto - CANESTRARI Stefano - MANNA Adelmo - PAPA Michele, Milano, UTET giuridica, 2019, p. 1179-1212 
MANES Vittorio, L'oracolo algoritmico e la giustizia penale: al bivio tra tecnologia e tecnocrazia, in Intelligenza artificiale. Il diritto, i diritti, l'etica, a cura di RUFFOLO Ugo, Milano, 2020, p. 547-567

MELCHIONDA Achille, Prova in generale (diritto processuale penale), in Enc. dir., Agg., I, Milano, Giuffrè, 1997, p. 838-865

MESSINA Salvatore, Il regime delle prove nel nuovo codice di procedura penale, Milano, SEL, 1914

MITTERMAIER C.G.A., Teoria della prova nel processo penale (1834), trad. it., Milano, Sanvito, 1858

MURA Alberto, Teoria bayesiana della decisione e ragionevole durata del processo, in Cass. pen., 2007, p. 3104-3116

NIEVA-FENOLL Jordi, Intelligenza artificiale e processo (2018), trad. it., Torino, Giappichelli, 2019

NOBILI Massimo, sub art. 187, in Commento al nuovo codice di procedura penale, coordinato da Chiavario Mario, II, Torino, UTET, 1990, p. 389-395

NUVOLONE Pietro, Le prove vietate nel processo penale nei paesi di diritto latino, in Riv. dir. proc., 1966, p. 442-475

PAULESU Pier Paolo, Giudice e parti nella "dialettica" della prova testimoniale, Torino, Giappichelli, 2002

PERELMAN Ch., La preuve en droit, essai de synthèse, in La preuve en droit, a cura di PERELMAN Ch. - FORIERS Paul, Bruxelles, Bruylant, 1981, p. 357-364

PRETI Giulio, Praxis ed empirismo, Torino, Einaudi, 1957

PUGLIATTI Salvatore, Conoscenza, in Enc. dir., IX, Milano, Giuffrè, 1961, p. 45-131

QUATTROCOLO Serena, Quesiti nuovi e soluzioni antiche? Consolidati paradigmi normativi vs rischi e paure della giustizia digitale "predittiva", in Cass. pen., 2019, p. $1748-1765$

RENZETTI Silvia, La prova scientifica nel processo penale: problemi e prospettive, in Riv. dir. proc., 2015, p. 399-423

RIVELLO Pier Paolo, La prova scientifica, in Trattato di procedura penale, diretto da UBERTIS, Giulio; VOENA, Giovanni Paolo, XVIII, Milano, Giuffrè, 2014

SABATINI Giuseppe, Prova (diritto processuale penale e diritto processuale penale militare), in Nss. D.I., XIV, Torino, UTET, 1967, p. 300-322 
SCARPELLI Uberto, Contributo alla semantica del linguaggio normativo [1959], Milano, Giuffrè, 1985

SIMEONE Giuseppe, Machine Learning e tutela della Privacy alla luce del GDPR, in Diritto e intelligenza artificiale, a cura di ALPA Guido, Pisa, Pacini, 2020, p. 275-295 SIRACUSANO Delfino, Studio sulla prova delle esimenti, Milano, Giuffrè, 1959 TAMBURRINI Guglielmo, Etica delle macchine. Dilemmi morali per robotica e intelligenza artificiale, Roma, Carocci, 2020

TARUFFO Michele, La prova dei fatti giuridici. Nozioni generali, in Trattato di diritto civile e commerciale, già diretto da CICU Antonio - MESSINEO Francesco e continuato da MENGONI Luigi, III, 2, 1, Milano, Giuffrè, 1992

TARUFFO Michele, Prova giuridica, in Enc. dir., Ann., I, Milano, Giuffrè, 2007, p. $1016-1037$

TARUFFO Michele, Studi sulla rilevanza della prova, Padova, CEDAM, 1970

UBERTIS Giulio, Fatto e valore nel sistema probatorio penale, Milano, Giuffrè, 1979

UBERTIS Giulio, Il giudice, la scienza e la prova (2011), in ID., Argomenti di procedura penale, IV, Milano, Giuffrè, 2016, p. 25-39

UBERTIS Giulio, Intelligenza artificiale, giustizia penale, controllo umano significativo, in Sistema penale, 11 novembre 2020, p. 1-15, consultabile all'indirizzo internet https://sistemapenale.it

UBERTIS Giulio, La prova penale. Profili giuridici ed epistemologici, Torino, Giappichelli,1995

UBERTIS Giulio, Profili di epistemologia giudiziaria, Milano, Giuffrè, 2015

UBERTIS Giulio, Prova: II) teoria generale del processo penale, in Enc. giur. Treccani, Agg., XVII, Roma, 2009, p. 1-14

UBERTIS Giulio, Prova scientifica e giustizia penale, in Riv. it. dir. proc. pen., 2016, p. 1192-1203

UBERTIS Giulio, Quaestio facti e quaestio iuris, in Quaestio facti, 2020, n. 1, p. 67-74, consultabile all'indirizzo internet https://www.quaestiofacti.com

UBERTIS Giulio, Ricostruzione del sistema, giusto processo, elementi di prova, in ID., Sisifo e Penelope. Il nuovo codice di procedura penale dal progetto preliminare alla ricostruzione del sistema, Torino, Giappichelli, 1993, p. 263-271 
UBERTIS Giulio, Sistema di procedura penale, I, Principi generali, Milano, Giuffrè, 2017

VERDE Giovanni, Prova in generale: b) teoria generale e diritto processuale civile, in Enc. dir., XXXVII, Milano, Giuffrè, 1988, p. 579-648

\section{Additional information and author's declarations (scientific integrity)}

Conflict of interest declaration: the author confirms that there are no conflicts of interest in conducting this research and writing this article.

Declaration of authorship: all and only researchers who comply the authorship requirements of this article are listed as authors; all coauthors are fully responsible for this work in its entirety.

Declaration of originality: the author assures that the text here published has not been previously published in any other resource and that future republication will only take place with the express indication of the reference of this original publication; he also attests that there is no third party plagiarism or self-plagiarism.

\section{Dados do processo editorial}

(http://www.ibraspp.com.br/revista/index.php/RBDPP/about/editorialPolicies)

- Recebido em: 01/02/2021

Equipe editorial envolvida

- Editor-chefe: 1 (VGV)

Autor convidado - invited author

- Editoras-associadas: 2 (KK e BC) 
HOW TO CITE THIS ARTICLES (ABNT BRAZIL):

UBERTIS, Giulio. I criteri di ammissibilità probatoria. Revista Brasileira de Direito Processual Penal, Porto Alegre, vol. 7, n. 1, p. 189-214, jan./ abr. 2021. https://doi.org/10.22197/rbdpp.v7i1.517

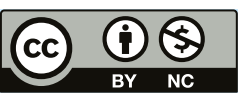

Esta obra está licenciada com uma Licença Creative Commons Atribuição-NãoComercial 4.0 Internacional. 